University of Wollongong

Research Online

Faculty of Engineering and Information

Faculty of Engineering and Information

Sciences - Papers: Part A

Sciences

$1-1-2011$

Concrete digital computation: What does it take for a physical system to compute?

Nir Fresco

University of New South Wales, nfresco@uow.edu.au

Follow this and additional works at: https://ro.uow.edu.au/eispapers

Part of the Engineering Commons, and the Science and Technology Studies Commons

Research Online is the open access institutional repository for the University of Wollongong. For further information contact the UOW Library: research-pubs@uow.edu.au 


\title{
Concrete digital computation: What does it take for a physical system to compute?
}

\begin{abstract}
This paper deals with the question: what are the key requirements for a physical system to perform digital computation? Time and again cognitive scientists are quick to employ the notion of computation simpliciter when asserting basically that cognitive activities are computational. They employ this notion as if there was or is a consensus on just what it takes for a physical system to perform computation, and in particular digital computation. Some cognitive scientists in referring to digital computation simply adhere to Turing's notion of computability. Classical computability theory studies what functions on the natural numbers are computable and what mathematical problems are undecidable. Whilst a mathematical formalism of computability may perform a methodological function of evaluating computational theories of certain cognitive capacities, concrete computation in physical systems seems to be required for explaining cognition as an embodied phenomenon. There are many non-equivalent accounts of digital computation in physical systems. I examine only a handful of those in this paper: (1) Turing's account; (2) The triviality "account"; (3) Reconstructing Smith's account of participatory computation; (4) The Algorithm Execution account. My goal in this paper is twofold. First, it is to identify and clarify some of the underlying key requirements mandated by these accounts. I argue that these differing requirements justify a demand that one commits to a particular account when employing the notion of computation in regard to physical systems. Second, it is to argue that despite the informative role that mathematical formalisms of computability may play in cognitive science, they do not specify the relationship between abstract and concrete computation.
\end{abstract}

\section{Keywords}

digital, compute, computation, does, take, physical, concrete, system

\section{Disciplines}

Engineering | Science and Technology Studies

\section{Publication Details}

Fresco, N. (2011). Concrete digital computation: What does it take for a physical system to compute? Journal of Logic, Language and Information, 20 (4), 513-537. 


\title{
Concrete Digital Computation: what does it take for a physical system to compute?
}

\author{
Nir Fresco \\ School of history \& philosophy, University of New South Wales, Sydney, Australia \\ Fresco.Nir@Gmail.com
}

\begin{abstract}
This paper deals with the question: what are the key requirements for a physical system to perform digital computation?

Time and again cognitive scientists are quick to employ the notion of computation simpliciter when asserting basically that cognitive activities are computational. They employ this notion as if there was or is a consensus on just what it takes for a physical system to perform computation, and in particular digital computation. Some cognitive scientists in referring to digital computation simply adhere to Turing's notion of computability. Classical computability theory studies what functions on the natural numbers are computable and what mathematical problems are undecidable. Whilst a mathematical formalism of computability may perform a methodological function of evaluating computational theories of certain cognitive capacities, concrete computation in physical systems seems to be required for explaining cognition as an embodied phenomenon.
\end{abstract}

There are many non-equivalent accounts of digital computation in physical systems. I examine only a handful of those in this paper: 1. Turing's account; 2. The triviality "account”; 3. Reconstructing Smith's account of participatory computation; 4. The Algorithm Execution account. My goal in this paper is twofold. First, it is to identify and clarify some of the underlying key requirements mandated by these accounts. I argue that these differing requirements justify a demand that one commits to a particular account when employing the notion of computation in regard to physical systems. Second, it is to argue that despite the informative role that mathematical formalisms of computability may play in cognitive This is a preprint of the article appearing in the Journal of Logic, Language and Information. It is reproduced with the kind permission of Springer-Verlag. The final publication is available at http://link.springer.com/article/10.1007/s10849-0111-9147-8. A more recent version appears in Physical Computation \& Cognitive Science (http://link.springer.com/book/10.1007/978-3-642-41375-9). 
science, they do not specify the relationship between abstract and concrete computation.

\section{Introduction}

Oftentimes, cognitive scientists are quick to employ the notion of computation simpliciter when asserting basically that cognitive activities are computational. Unfortunately, it seems all too often that a clearer understanding of computation is distorted by philosophical concerns about cognition. In this respect I am sympathetic to David Israel's claim that computation proper (rather than just computability) has mostly been disregarded by cognitive science. This, he argues, could be explained mostly by the reluctance to look at computation "free of any admixture of direct concerns with issues in... cognitive science" (Israel 2002: p. 181).

Some researchers in referring to digital computation simply adhere to Alan Turing's notion of computability when attempting to explain cognitive behaviour. He modelled the behaviour of human mathematicians in performing paper and pencil calculations. So the motivation for cognitive science adopting the Turing machine (henceforth TM), rather than other formalisms of computability, is clear. Still, classical computability theory studies what functions on the natural numbers are computable, and not the spatiotemporal constraints that are inherent to cognitive phenomena.

Any analysis of cognitive phenomena, which is based solely on mathematical formalisms of computability, is at best incomplete. It has been proven that Emil Post's machines, Stephen Kleene's formal systems model, Kurt Gödel's recursive functions model, Alonzo Church's lambda calculus, Shepherdson and Sturgis' Register machines and TMs - are equivalent. They all identify the same class of functions, in terms of the sets of arguments and values that they determine, as computable (Kleene 2002: pp. 232-233; Parikh 1998; Soare 
1996: p. 298). Indeed, computation (broadly interpreted) need not always be viewed as computation in physical systems. The above mathematical formalisms can exist in a Platonic realm and be independent of any particular physical implementation.

Whilst they could be useful as mathematical tools for evaluating the plausibility of a computational level analysis of a particular cognitive capacity, they are of the wrong kind for explaining cognition as an embodied phenomenon. Concrete computation in physical systems seems to be a more appropriate candidate for the job ${ }^{1 \cdot}$ It is not in vain that the reigning trends in contemporary cognitive science (whether it be connectionism or dynamicism) emphasise the embeddedness and embodiment of cognitive agents. This is one motivation for examining extant accounts of concrete computation, before we can make any sense of talk about 'cognitive computation', 'neural computation' or 'biological computation'.

Furthermore, even the notion 'computation' could be used to denote several classes of computation. The traditional divide of computation into digital and analogue may be insufficient with the introduction of connectionist computation, quantum computation, hypercomputation and so on. Some recommend using the broader notion of 'generic computation' to be all encompassing and include all these different classes of computation (Piccinini \& Scarantino 2011: pp. 10-12). These distinctions will not be addressed here, rather my focus will be on digital computation only. In order to avoid confusion, hereafter the term 'computability' will be used to refer to the mathematical notion of digital computability; and the term 'computation' will be used to refer to concrete digital computation as it is actualised in physical systems.

There are many non-equivalent accounts of digital computation in physical systems on offer. Amongst those are TMs, formal symbol manipulation, physical symbol systems,

\footnotetext{
${ }^{1}$ For the purposes of this paper, I shall remain neutral on whether cognition can indeed be fully explained computationally. This question can remain unanswered for now.
} 
algorithm execution, information processing, and the list continues. I examine only a handful of those in this paper. 1. Turing's account identifies the following key requirements: having the capacity to recognise symbols, having the capacity to write symbols and store them in memory, having the capacity to change states; and having the capacity to follow instructions ${ }^{2}$. 2. The triviality "account" suggests that any (sufficiently) complex physical system performs computation. 3. A reconstruction of Smith's account of participatory computation entails that the key requirements are the following: the situatedness of the computing system, computational processes extending beyond the physical boundaries of the system, and the system standing in semantic relations to distal states of affairs. 4. The Algorithm Execution account identifies not only the execution of algorithms as the key requirement, but also the existence of the right labelling scheme, a formal description of that system, and an "honest" model that binds them together.

No novel account of computation is offered here. The motivation for this paper is to examine the conflict among well-known accounts and argue that they entail sufficiently distinct requirements for a physical system to compute to justify the demand that one commits to a particular account when employing the notion of concrete computation. Whilst the focus here is on cognitive science, this demand is unbiased. It applies just as well to biologists, astronomers and anyone else that employs 'computation' as explanans for some phenomenon. My first goal is to identify and clarify some of the underlying differences between these accounts by considering their key requirements. The second goal is to argue that despite the informative role that mathematical formalisms of computability may play in cognitive science, they do not specify the relationship between abstract and concrete computation.

\footnotetext{
${ }^{2}$ Strictly speaking, whereas Universal TMs (hereafter, UTMs) follow instructions, special purpose TMs need not be construed as following instructions in an explicit sense. The distinction between TMs and Universal TMs is further discussed below.
} 


\section{Turing's account}

\subsection{TMs from mathematical and physical perspectives}

Turing was the first one to formulate a comprehensive theory of computability. Turing (1936) analysed computability primarily as a mathematical concept, namely what functions on the natural numbers are computable, but also from a mechanical standpoint: what can be done by mechanically following a procedure. Although, he analysed (mathematical) computability, rather than (concrete) computation, his account must be examined as the basis for any possibly adequate account of concrete computation. He achieved a metamathematical proof that the reiteration of some basic atomic operations is sufficient for all the operations of mathematics at large (Agassi unpublished). In that respect, Post's machines, Kleene's formal systems, Gödel's recursive functions, and Church's lambda calculus also identify the same class of functions as computable.

Still, these formalisms of computability are not examined under my analysis of Turing's account. They are subdivided (amongst other things) in terms of algorithmic versus nonalgorithmic models. Gödel's recursive functions, for one, are essentially non-algorithmic. From a strictly intensional viewpoint, recursive functions and recursion theory do not analyse anything about what computers can or cannot accomplish at all (Soare 1996: p. 307). Recursive functions could also exist in a Platonic realm where mathematical objects exist outside space and time. On the other hand, TMs and Church's lambda calculus have an algorithmic interpretation inherent to the computation of $F(x)$. Despite being of great philosophical importance how such different formalisms can be equivalent, this question is not addressed here.

Abstract TMs could be described as follows (Turing 1936: pp. 231-232).

- The machine has a head (a read/write device going along the tape) that is only capable 
of a finite number of "conditions" $\left(\mathrm{q}_{1}, \mathrm{q}_{2}, \ldots, \mathrm{q}_{\mathrm{n}}\right)$, which are called "m-configurations" and constitute the machine's states. This latter part is Turing's finiteness condition on the number of states (Soare 1996: p. 292).

- The machine is supplied with an unlimited running tape, which is divided into squares and constitutes the machine's memory

- At any given time there is only one scanned square, which may bear a symbol (referred to as a scanned symbol). This is Turing's finiteness condition on the number of symbols scanned at any given moment (Soare 1996: p. 292)

- The behaviour of the machine is determined by an ordered pair consisting of its state and the symbol scanned

- The machine can write a new symbol after erasing any existing scanned symbol

- The machine may also change the square it is scanning by shifting it one place to the right or left

- The machine may change its state

It is important to emphasise that from a mathematical perspective a TM is an abstract mathematical object that may be formally defined by the following 7-tuple (Hopcroft 2001: p. 319). $\mathrm{TM}=\left(\mathrm{Q}, \Sigma, \Gamma, \delta, \mathrm{q}_{0}, \mathrm{~B}, \mathrm{~F}\right)$ where:

- Q stands for the finite set of states of the TM.

- $\Sigma$ stands for the finite set of input symbols

- $\Gamma$ stands for the complete set of tape symbols and is a superset of $\Sigma$

- $\delta$ stands for the transition function

- $\mathrm{q}_{0}$ stands for the initial state and is a member of $\mathrm{Q}$

- B stands for a blank symbol

- F stands for the set of final states 
So, from a mathematical point of view it is an abstract transition function that can exists in the Platonic realm. The TM was originally introduced as a model of a human computer (or mathematician) engaged in a paper and pencil calculations. It is figuratively described as a machine that reads and writes symbols in the process of computing that function. But it is "no more a machine... than a model aeroplane is an aeroplane, although like any model, its theoretical utility lies in the fact that it has certain properties that stand in a systematic relationship to the things it models" (Seligman 2002: p. 210, italics added).

Although a TM is an idealisation of a machine, it is a simple model of a digital computer on which all real modern computers are based ${ }^{3}$. This model was so simple as to facilitate building actual instances according to this design (Israel 2002: p. 193). Turing's analysis does not take into account important physical constraint considerations: a TM exerts no energy ${ }^{4}$, it never breaks down and has an infinite tape. It was acceptable in the discourse on what is computable, in principle by humans or machines to disregard such constraints. Abstract ideas, such as Turing's, can often be developed in detail and acquire some empirical aspects and win scientific status (Agassi unpublished). And indeed, the four requirements implied by his abstract $\mathrm{TMs}^{5}$ should be viewed as regulative principles and be supplemented to ensure that physical (computing) systems obey the laws of physics (as attempted by Robin

\footnotetext{
3 Admittedly, the later Register Machine formalism seems a more adequate model of the modern digital computer, which manipulates data and instructions stored in registers, than the TM, which has to scan the data back and forth along its tape (Soare 1996: p. 298).

${ }^{4}$ And yet moving its head to the left or right, reading and writing a symbol on a square and so on are all physical actions with causal effects.

${ }^{5}$ Whilst TMs or UTMs are conventionally used to model digital computation, Robert Soare (2009: p. 387) argues that the Oracle TM should be used to model online digital computation. An offline computing process is defined as one that begins with a program and some input, and proceeds internally without any interaction with an external device. An online computing process is one that interacts with its environment. UTMs and special purpose TMs lack this online capacity, for they begin with a fixed program and fixed input and proceed without further external input until they halt. The Oracle TM, in contrast, is typically a fixed program at the core, and the Turing type oracle is the mechanism for communicating with the environment (e.g., a database). Be that as it may, modern digital computers do not typically invoke any non-algorithmic "oracle" processes that guess the right answers. Martin Davis (1958: pp. 20-24) proposes a different model of an ordinary TM, in the context of relative computability, that can interact with the external world.
} 
Gandy).

\subsection{The key requirements according to Turing's account}

According to Turing (1936: pp. 231-232) the key requirements for a physical system to perform digital computation are the following: 1 . having the capacity to recognise symbols; 2 . having the capacity to write symbols and store them in memory; 3 . having the capacity to change states; and 4. having the capacity to follow instructions.

The first fundamental requirement of Turing's account is the system having the capacity to recognise symbols ${ }^{6}$. Any computation executed by the machine cannot proceed without it being capable of identifying the scanned symbol and responding correctly (erasing a symbol, writing a new one etc.) based on that specific symbol. The Turing scanner moves back and forth through the tape and scans finitely many basic symbols (Turing's finiteness condition on the number of symbols, Soare 1996: p. 292). If the TM used infinitely many symbols, then some of those symbols might be too similar to the others to be reliably distinguished from them in finite time (Parikh 1998). And without the capacity to recognise the scanned symbol its operation will be completely pointless. A TM unable to recognise symbols will not be able to read symbols off the tape rendering it incapable of successfully completing its operation.

More specifically, this requirement may be broken down further into three conditions, which were proposed by Raymond J. Nelson. He listed four conditions that an FSA has to satisfy to count as accepting, and the first three seem to be essential for symbol recognition (Nelson 1982: pp. 166-170). The first condition, universality and discrimination, is that the automaton must be able to assign types to tokens (or universals to particulars) over a potentially infinite domain. If an FSA can accept a particular symbol, it must be able to

\footnotetext{
${ }^{6}$ Yet nothing essential hinges on TM's symbols being symbols. The only relevant property of the symbols is that they are determined by perceivable properties (i.e., be recognisable) of the squares and can be modified by the agent (Seligman 2002: pp. 217-218).
} 
discriminate it among many types over a very large (though finite) domain. Secondly, the One-Token-Many-Type condition states that the acceptance relation be merely relational rather than functional, namely that the FSA should be able to assign a token to more than one type. Thirdly, the One-Type-Many-Tokens condition states that the FSA must be able to accept the elements of a single type from any one of several disjoint sets of tokens ${ }^{7}$. His fourth condition requires a token to be recognised, although its individuating properties are degraded, by some means of correction based on the context etc.

Moreover, Turing's second key requirement is the system having the capacity to write symbols and store them in memory. A TM reads symbols, but in order to perform non-trivial computations it also writes symbols for intermediate calculations and printing the output. The machine's tape is a general-purpose storage, which serves both as a long-term working memory and the input/output device ${ }^{8}$. Without the capacity to store symbols in memory, TMs would reduce to finite state automata (hereafter FSA). For a TM is an FSA with a single infinite tape for reading and writing symbols (Hopcroft et al 2001: p. 317). In the above 7tuple notation of a TM, only $\Gamma$ and $\mathrm{B}$ are elements added to the 5-tuple notation of an FSA and the transition function is of the type: $\mathrm{Q} \times \Gamma \rightarrow \mathrm{Q} \times \Gamma \times\{\mathrm{L}, \mathrm{R}\}$ (where $\mathrm{L}, \mathrm{R}$ stand for moving one cell left or right on the tape) (Israel 2002: p. 193). Although FSA do compute, their computational power is limited compared with that of TMs and UTMs, due to their lack of a storage device (Wells 1998: 287).

Furthermore, according to the third requirement a TM could change its state or remain in its current state in accordance with three following neighborhood conditions. Firstly, the

\footnotetext{
${ }^{7}$ Unlike the first condition, the third one implies that there is a family of disjoint sets and one and the same type is the type for every set of that family.

${ }^{8}$ TMs must have input for their normal operation, but they do not necessarily produce an output. In the latter case they accept the input, perform some operation and halt. Interestingly, a TM need not have a single tape. It can have multiple tapes with a finite sequence of input symbols on one tape. Single-tape TMs are equivalent to multi-tape TMs: any multi-tape TM can be simulated by a single-tape TM (Hopcroft et al 2001: pp. 336-338).
} 
TM can change the symbol only in a scanned square and then at most one symbol. Secondly, the TM can move to a different set of scanned squares, but only within a certain bounded distance of a scanned square. Thirdly, any atomic operation must depend only on the TM's current state and the symbol in the scanned square (Israel 2002: p. 189; Soare 1996: p. 292). The TM may be in any of a finite number of states formally denoted above by Q. The machine could change its state as a function of its current state and the symbol just scanned. However, the next state of the TM may also be the same state it was in. Again, this requirement too is a characteristic of FSA, which either change their state or not depending on the symbol read.

Finally, the fourth requirement is the system having the capacity to follow instructions. A TM acts in accordance with a table of instructions (or a program), although Turing did not explicate the mechanism that causes the machine to follow these instructions. But in the sense that TMs are merely idealisations, it was not essential that he explicate such a specific mechanism, which causes the machine to follow these instructions. An important distinction needs to be made between special purpose TMs and UTMs. Whereas TMs can only be construed as acting in accordance with instructions, which are hardwired (or implicit), UTMs also follow instructions explicitly.

The capacity to follow instructions (or execute a program) is maximised in the UTM. It is able to carry out every computation that can be carried out by any special purpose TM. The UTM is the first conceptual stored-program ${ }^{9}$ general-purpose digital computer: the control of the computer's operation is achieved by storing an encoded program into its memory

\footnotetext{
${ }^{9}$ Gualtiero Piccinini (2008b: pp. 55-56) argues that UTMs should not be deemed stored-program computers contrary to the received view, since the machine's tape is used as both an input/output device and a memory. Also, he adds that Turing originally considered the machine's internal state, rather than its tape, a memory component. The TM's internal states are merely short-term memory, and not long-term working memory that is required for a stored-program computer.
} 
(Copeland 2003: pp. 5-6). The machine's program allows it to perform different sequences of operations and yields a powerful kind of computing systems, namely programmable systems.

\section{The triviality "account"}

According to the triviality "account" every physical object performs digital computation. There is no fact of the matter about what digital computation is, and thus everything can be interpreted to perform some digital computation. The two main proponents of this view are John Searle and Hilary Putnam. Searle (1990) claims that even his wall can be interpreted to implement the (now obsolete) WordStar program. Putnam (1988: pp. 121-125) claims that every physical object is a realisation of every abstract FSA or a program. In other words, every physical object performs any particular computation. This "account" is not an account of concrete computation, but rather a consequence of both Putnam's state-transition account and Searle's criticism of computationalism. It is examined here for completeness and to resist conspicuous attempts to trivialise digital computation. If everything physical computes, concrete computation ceases to be interesting in its own right. But it is far from being trivial.

\subsection{The interpretative characterisation of theories of computation}

On Searle's view, the characterisation of a process as computational is an external knower-dependent characterisation. Searle (1990) asserts that some alternative definitions of digital computation (as those suggested by Brian Cantwell Smith) emphasise (arguably important) features, such as causal relations between program states, programmability, controllability of the computing system and its situatedness in the real world. However, he argues that even these restricted definitions (without addressing them directly) are not useful 
in overcoming the central challenge of syntax being essentially a knower relative notion ${ }^{10}$. There is no fact of the matter about what constitutes a process being computational; this depends on an interpretation external to the process, since it is not intrinsic to the process. On his view, computational states of an object are not discovered within its physics, but rather they are assigned to it. So any theory of digital computation would be necessarily interpretative.

Accordingly, there is no fact of the matter about what constitutes computation. Searle (1990) argues that on a standard interpretation of digital computation, for any object at all there is some description of that object such that under that description it performs digital computation. Further, for any program $P$, there is some sufficiently complex object such that there is some description of that object under which it can be deemed to implement $P$. He asserts that if a physical object has some underlying microscopic causal pattern in it that is isomorphic with the formal structure of $P$, then that object implements $P$. Thus, on his view, it follows that a big enough wall, for instance, can be deemed to implement the WordStar program, because it has some pattern of molecule movements that is isomorphic with the formal structure of WordStar.

\subsection{Putnam's state-transition account}

In a similar vein, Putnam (1988: p. 121) argues that every ordinary open system is a realisation of every abstract FSA. 'Ordinary open system' refers to systems that are open to influences such as gravitational and electromagnetic forces that result in those systems constantly being subject to change. In an attempt to criticise computationalism, Putnam is set to show that all systems, which exhibit the same behaviour, implement the same program. So

\footnotetext{
${ }^{10}$ Searle argues that computation is observer-relative, however, I choose to replace observer with knower to avoid some potential epistemic implications. Rather than committing to an empiricist view, the knowledge of a knower could be based on reason, sense-experience or both. This does not weaken Searle's position regarding computation being relative to rational agents (humans, Martians or dolphins).
} 
if computationalism were true, it would render (the once popular) behaviourism true (ibid: pp. xi, 124-125). To advance his arguments, Putnam (1988: p. xv) identifies computational description with functional organization and proves the theorem according to which in a sense everything has every functional organization and thus also every computational description.

As the basis of his theorem, Putnam puts forward two principles that are crucial to his proof: the principle of continuity and the principle of non-cyclical behaviour.

1 "The principle of continuity. The electromagnetic and gravitational fields are continuous, except possibly at a finite or denumerably infinite set of points" (ibid: p. 121).

2 "The principle of non-cyclical behavior. The system $\mathrm{S}$ is in different maximal states at different times. This principle will hold true of all systems that are not shielded from electromagnetic and gravitational signals from a clock" (ibid).

According to the first principle, which assumes classical physics (ibid), the electrical and gravitational fields are continuous. And according to the second one, every ordinary open system is in different (maximal) states at different times. For Putnam, the maximal state of a system is its overall state that describes the system's physical composition. In classical physics, a maximal state is the value of all the relevant parameters of the physical system inside the boundaries of the system at any given time (Buechner 2008: p. 137). The second principle ensures that there is no ambiguous mapping (i.e., non one-to-one) of the physical states of a system to its computational states. The natural clock constantly stamps the system in such a way that the system cannot have a duplicated physical state and enter the same maximal state twice.

On Putnam's view, there is no metaphysical need for a knower to attribute a computational description to a physical system. Instead, he proves the theorem that every physical object is a realisation of every program. To accomplish that, Putnam (1988: pp. 122- 
123) demonstrates that any physical system could realise any arbitrary FSA. He accepts that a causal relation must be established between his construed computational states A and $\mathrm{B}$. Putnam asserts that the system being in A and constrained by its boundary conditions (when it is in A) causes the system to move to state B. Given the state of the boundary of the system at time ti, then it follows from his lemma (ibid: pp. 121-122) and the principle of continuity, that the inner part of the system has to change from one state it was at time ti-1 to a distinct state. Thus, the transitions between A and B are causal (Chrisley 1995: p. 409). Since a computational state $A$ is mapped to physical state space $S_{1}$ and a computational state $B$ is mapped to physical state space $\mathrm{S}_{2}$, it follows that the system is either in state A or B at the appropriate time.

\subsection{The key requirements for a system to perform digital computation}

According to Searle (1990), the key requirements are the existence of a knower and the system being a sufficiently complex physical object. The former requirement is a metaphysical one and Searle proposes a simple criterion to put it to the test: suppose that there were no knowers, would there be any physical systems performing digital computations? His answer is no, in a similar way that chairs would not exist except relative to some knowers, who regarded them as such. Strictly speaking, chairs and other physical objects would still exist; only no one would interpret them as functioning as chairs or performing digital computations. For Searle, digital computation cannot exist without the existence of knowers, who assign this feature to the system performing it. The broader consequence of his view is a binary divide of all phenomena into knower-relative features and intrinsic physical features. Digital computers, chairs and glasses are knower-relative, but molecular movements and gravitation are intrinsic physical features. The latter would still exist even in our absence.

Moreover, Searle's second requirement is of a physical nature, rather than a 
metaphysical one: it posits that the system has to be sufficiently complex. Searle (1990: p. 27) argues that for any computer program there is some sufficiently complex object and some description of that object under which it executes that program (thus performing a digital computation). But then he goes on saying that a "big enough wall" (ibid) would be implementing any program, because it has various causal patterns of molecule movements each of which is isomorphic with the formal structure of the program implemented. It follows that his second requirement reduces to some arbitrary molecule movements in the physical object coexisting with some arbitrary description. Any physical object consisting of a sufficiently large number of discernible parts could be said to implement any program under the appropriate description.

Putnam, on the other hand, does not subscribe to the metaphysical requirement, and makes Searle's second requirement stricter. Putnam makes two assumptions regarding ordinary open physical systems and the individuation of physical states. The first one is that a system cannot be assigned distinct computational states $\mathrm{A}$ and $\mathrm{B}$ at $\mathrm{t}_{1}$ and $\mathrm{t}_{2}$ respectively, if the corresponding physical states at $t_{1}$ and $t_{2}$ cannot be distinguished from one another. Under his interpretation of physical systems, if a system can be individuated into the number of distinct state necessary to implement any FSA, then it is sufficiently complex (Chrisley 1995: p. 411). His second assumption is that the physical states of those systems should be individuated by their relative intrinsic properties and not temporally. This is implied by insisting on the principle of non-cyclical behaviour that states that those systems are in different states at different times.

\section{Reconstructing Smith's account of participatory computation}

According to Smith (2002: p. 24; 2010: p. 38) there will never be a satisfactory and 
intellectually productive theory of computation. Nevertheless, in the following sections I propose an outline of a possible account of computation that could be reconstructed from Smith's view of participatory computation. This reconstructed account is based on the following key requirements:

1. The situatedness of the computing system;

2. The internal states of the computing system operating effectively, but computational processes also extending beyond the physical boundaries of the system;

3. The system standing in semantic relations to distal states of affairs.

Firstly, Smith (2002: p. 37; 2010: p. 17) argues that computers are concrete, situated entities that participatorily engage and can do real work in the material world. Computational systems like those, which underlie modern real-time embedded operating systems in packet routers that relay Internet traffic around the world or aircraft navigation systems that guide planes and pilots in the sky everyday, are as concrete and situated as it gets (Smith 2010: p. 9). Real-world computing systems ${ }^{11}$ are extended to include a variety of peripheral modules, which are controlled by the computer's operating system, such as keyboards, screens, printers, hardware cables, network cables and so on. These all function as transducers through which the computing system actively interacts with the world that indiscriminately includes it (Smith 2002: p. 37). The traditional physical boundaries of a computer do not block it from interacting with the environment, in which it is situated, in such a manner that its internal operation is also dependent on input from that environment (Smith unpublished).

Interestingly, Smith's first requirement is also Andrew Wells' goal in arguing that it is

\footnotetext{
${ }^{11}$ Smith (1996: p. 69; 2002) advocates three evaluation criteria for computation. One of these criteria is the empirical criterion, which methodologically mandates that an adequate analysis must do justice to computational practice: real-world computing systems (for a critique of Smith's criteria see Fresco 2008).
} 
not a new paradigm of computation that is needed, but simply a better understanding of Turing's analysis. He advocates an externalist rather than an internalist interpretation of Turing's analysis of computation (Wells 1998: pp. 270-271). On his view, whilst the finite state controller is part of the TMs internal architecture, the memory/tape mechanism is deemed a part of the external environment. TMs are thus viewed as being irreducibly "world involving" (ibid: p. 280), fostering an interactive approach to concrete computation: computing systems are situated in and interact with the surrounding environment. Wells argues that Turing indeed thought of the machine's tape as instantiated in a medium that is different from the controller. This is because the human (computer) memory is limited by its supervenience on a finite substrate, whereas the TM's tape is infinite (ibid: p. 282).

Secondly, Smith (2010: pp. 29, 36) asserts that not only do internal states of a computing system operate effectively (roughly: they are causally influential); computational processes typically extend beyond the physical boundaries of the system. The first part of the requirement is true simpliciter for any physical process and computation is no different. Whether one examines computation as merely a process or considers its physical actualisation in a computing system, an efficacy relation obtains among the internal states of the process or the system. This is how a computational process moves from its initial state and input, through state-transitions to producing output and terminating at the final state. But the crucial point for Smith (1996: pp. 70-71) is that a computing system crosses the physical and semantic boundaries. The computing system involves causal interactions between and among symbols (internal) and referents (internal and possibly external ${ }^{12}$ ) (Smith 2010: p. 29). Even if we imposed theoretical boundaries between the system and the surrounding environment,

\footnotetext{
${ }^{12}$ A traffic monitoring computing system, for instance, is affected by and affects the traffic on the road (as an external referent). However, the Sun would be a distal external referent, which is not affected by or affects the computing system (unless the system is somehow engaged in absorbing heat or light from the Sun).
} 
these would not be boundaries to the flow of effect (Smith unpublished). For instance, a process, which sends a message across to another external system, will send a signal that "travels" beyond the input/output boundaries and await an acknowledgement response.

In other words, for Smith (2010: p. 36), real-world computing systems need to be analysed in the middle ground realm between the abstract and the concrete. The analysis required here is metaphysical and one that has not yet been recognised by the natural sciences. Computing systems need to be understood at a level more abstract than is traditional in classical physics, but also at a more concrete level than classical computability theory. According to Smith (1996: pp. 70-71; 2002: pp. 35-36), the physical boundary divides what is internal to the computing system (e.g., registers, CPU, memory etc.) from what is external (i.e., the environment in which it is embedded). The semantic boundary divides the realm of symbols being manipulated during a computation from their referents, which could be internal (e.g., instructions stored in the system's memory) or external (e.g., salaries and other states of affairs).

Although it is typically assumed that computation merely operates effectively on symbols, this is a mistake according to Smith (1996: pp. 70-71; 2002: pp. 34-35; 2010: pp. 28-29). He argues that computing systems are concrete representational systems, which stand in semantic relations to distal and other non-effective states of affairs. Computing systems cut across both boundaries where manipulated symbols do not just affect internal referents (for instance, a memory address and the instruction in the memory to be executed next), but also external referents (for example, a numeral stored in the payroll database and a corresponding numeric value of an employee's salary). And this crossing of the semantic boundary makes the computing system an intentional one, unlike a wall, for example. When a symbol is manipulated causing a numeric value in the payroll database to change, all going well, this 
will lead to an employee getting a pay rise (or less fortunately, a pay cut) in the real world.

Moreover, the efficacy that constrains computation in computing systems has direct consequences on the real world. Smith (2010: pp. 23, 29) argues that computing systems embodied in the real world are actively involved in their subject matters, in being consequential players in the very world they represent. In their operation they make effective use of and affect the very states of affairs that their symbols semantically represent. Even a simple example of a loop counter that returns as output the number of unique (type) elements that were entered as input demonstrates according to Smith (1996: p. 71) the crossing of the semantic boundary. The numeral ' 3 ' will be returned as output, for instance, given an input $<$ 'dog', 'cat' 'mouse', 'dog'> following a series of effectively related computational steps. A long downtime of a backbone mail-server, or an auto-navigated vehicle losing its set course, exemplify how local internal states of computing systems have long-distance correlations to some states of affairs by means of local causal efficacy (Smith 2002: p. 37; 2010: p. 36).

\section{The Algorithm Execution account}

According to this account concrete digital computation amounts to execution of algorithms. Jack Copeland and Robert Cummins share this common view, although there are some key differences between their interpretations of executing an algorithm ${ }^{13}$. Copeland proposes a much more rigorous account of digital computation, which excludes noncomputing systems such as walls, clocks and planetary systems, but includes digital computers, TMs and finite state automata. Cummins' (1977: pp. 279-284; 1989: pp. 91-92; 1996: p. 610) account implies that for a system to perform digital computation it needs to execute an algorithm where its output and final state are a causal outcome of its input and

\footnotetext{
${ }^{13}$ For Cummins (1989: p. 91) to compute a function reduces to executing a program. But his analysis and his analogies to cooking recipes imply that for him an algorithm and a program are of the same kind.
} 
initial state. Copeland's (1996) account introduces further requirements: the existence of the right labelling scheme as well as a formal description of that system, and an "honest" model that binds them together.

On Cummins' (1989: pp. 89-92) interpretation, execution of an algorithm reduces to a disciplined step satisfaction, which can be given a systematic semantic interpretation. A system computes if the underlying steps of the algorithm are satisfied in a disciplined manner, which is determined by the appropriate causal connections that obtain between the steps and the system. For Cummins, (1989: p. 92; 1996: p. 10) computable functions that are satisfied by a process $P$ executed in system $S$ specify causal connections between steps in $P$ and between the final state of $S$ and its initial state. If $P$ satisfies functions $f$ and $g$ and $f$ 's output is $g$ 's input, then the satisfaction of step $f$ will yield the satisfaction of step $g . S$ is computing the addition function when the underlying steps it satisfies could be systematically interpreted as addition. A function lacking two arguments, which could be interpreted as addends, cannot be systematically interpreted as an addition function, for instance.

However, Copeland (1996: p. 353) argues that Cummins' analysis of computation is too narrow and falls prey to Searle's trivialisation of digital computation. He claims that a causal analysis of digital computation fails to encapsulate abstract systems like TMs or finite state automata, which are paradigmatic computing systems. For instance, in a virtual TM, which is simulated on a digital computer, no causal relations obtain between the contents of the virtual machine's tape and its actions. Yet, the virtual TM computes. Furthermore, it is possible to show that on some formal description of Searle's wall, the label-bearing states satisfy the appropriate functions, and so the wall computes!

Consequently, Copeland proposes a stricter account of digital computation by adding auxiliary requirements. On his view, a system $S$ would be computing a function $f$ iff there 
exists a labelling scheme $L$ and a formal description SPEC of an architecture and an algorithm specific to that architecture such that the pair $(S, L)$ is an honest model of SPEC. The labelling scheme designates certain parts of $\mathrm{S}$ as label bearers and provides the method for specifying the label borne by each label-bearing part at any given time. Copeland (1996: p. 338) exemplifies this labelling scheme by a pair of flip-flops where the voltage across the first one is $600 \mathrm{mV}$ and across the second one is $100 \mathrm{mV}$. This pair may be labelled $(1,0)$ and accordingly be described as representing the number two in a binary format. Importantly, an adequate labelling scheme L should not introduce any unintended temporal specifities into the theory. $\mathrm{L}$ should obtain for any $\mathrm{T}_{\mathrm{i}}$ and remain applicable throughout the lifetime of the computing system. But a labelling scheme, which only applies for $T_{1}$ to $T_{n}$ and does not specify what labelling holds prior to $T_{1}$ or subsequent to $T_{n}$, is incomplete (ibid: pp. 348-349).

Moreover, SPEC is a formal description of a certain functional architecture of the system $\mathrm{S}$ and algorithm, which is specific to this architecture ${ }^{14}$. Whilst algorithms are typically thought of as independent of the underlying machine that executes them, this dependency hinges on the primitive operations that are supported by this specific architecture. If, for instance, the algorithm is such that it sums two addends and saves the result in another data structure, then the underlying architecture should have at least two registers. For Copeland, this architecture could be either the concrete or conceptual structure of the computing system (Copeland, personal communication). Finally, the pair (S, L) is an honest model of SPEC when the labelling scheme is not ex post facto and the interpretation associated with that model supports counterfactuals about computation. For instance, if the computing system supports the ADD operation, then ADD must be part of SPEC even if the system never enters the state leading to the execution of this operation (ibid: pp. 341-342, 350-351).

${ }^{14}$ Copeland describes SPEC in terms of a set of axioms, but nothing of substance hangs upon this axiomatic method (1996: p. 338). 


\section{Discussion}

\subsection{Avoiding ambiguity about concrete digital computation}

My main argument proceeds as follows:

- (P1) There are many accounts of digital computation at our disposal.

- (P2) These accounts establish different (but not all irreducibly different) requirements for a physical system to perform digital computation.

- (P3) Therefore, extant accounts of computation are non-equivalent.

- (P4) Cognitive capacities are sometimes explained by invoking digital computation terminology.

- (P5) When employing an equivocal interpretation, one needs to commit to an explicit interpretation (or account).

- Therefore, one needs to commit to an explicit account of computation when explaining cognitive capacities by invoking digital computation terminology. Specifically, any computational thesis of cognition is unintelligible without a commitment to a specific account of computation.

The truth of the first premise is evident in the philosophical literature (cf. Piccinini 2007; Shagrir 1999; and Smith 2002, 2010). The accounts reviewed in this paper are only a handful. Others include the formal symbol manipulation account (Fodor 1975; Pylyshyn 1984), the physical symbol systems account (Newell and Simon 1976), Gandy’s account (1980) and the mechanistic account of computation (Piccinini 2007) ${ }^{15}$.

Similarly, premises four and five (at least) seem self-evident. Premise four is hardly

${ }^{15}$ For lack of space, not all accounts are examined here in detail. I discuss other accounts elsewhere (Fresco forthcoming). 
doubtable: computationalists take it for granted (Fodor 1975, Pylyshyn 1984, Newell \& Simon 1976, Marr 1982, van Rooij 2008) and so do some connectionists. Dynamicists do not subscribe to the computational theory of mind (Van Gelder \& Port 1995, Thelen \& Smith 1994), yet they reject it without committing to any particular account of computation proper. They reject it due to the presupposition that digital computation is inherently representational. I maintain that this presupposition is unjustified, for digital computation (not computationalism) could be explained without invoking any representational properties (barring internal representations) by appealing to causal or functional properties instead (see Fresco 2010 and Piccinini 2008a). As van Rooij (2008: p. 964) rightly points out, 'computation' and 'computationalism' have become associated with the symbolic tradition, but only sometimes with specific models in this tradition. Some accounts of concrete digital computation are indeed representational (cf. Smith's reconstructed account, Formal Symbol Manipulation, Physical Symbol Systems), but others need not be (cf. Copeland 1996, Chalmers 1995, Piccinini 2007). This simply reinforces the need to commit to a particular account of computation.

Moreover, premise five calls for disambiguation when there is an equivocation in terms. When some phenomenon is open to two interpretations or more, we should commit to one interpretation to avoid ambiguity. For instance, the concept depression has at least two typical meanings. In the sentence, "The great depression started in most countries in 1929 and lasted for a long time", it is clear that 'depression' means a long-term downturn in economic activity. On the other hand, in the sentence, "Long depression leads to making irrational decisions", 'depression' means something different. Analogously, when one asserts that hierarchical planning or linguistic tasks, for example, are computational, one ought to commit 
to a particular account of (digital) computation ${ }^{16}$. Is it in virtue of executing an algorithm, formally manipulating symbols, or implementing a TM that cognitive agents engage in hierarchical planning? Ambiguity may lead to poor communication, which may hinder the progress in the relevant research field, for it is clearly a collaborative effort.

Furthermore, the commitment to a particular interpretation should be consistent to avoid further ambiguity. From the two sentences above it follows that irrational decisions were made in the countries that suffered the great depression in 1929. This conclusion would only validly follow from its premises, if 'depression' has the same interpretation in both premises. Otherwise, whilst this conclusion may be plausible, it does not necessarily follow. This is also known as the fallacy of equivocation. Similarly, if one explains a particular cognitive capacity in virtue of an explicit account of concrete digital computation, one has to consistently adhere to that account. An explanation of a linguistic task in virtue of formal symbol manipulation and then in virtue of algorithm execution ceases to be a coherent story, since they are not equivalent (see premise three).

Prima facie, it might seem that premise two is self-defeating, but this is not the case. A possible consequence of all the requirements not being irreducibly different is some overlap between them. Thus, the requirements that are implied by one account could be reduced to some of the other requirements ${ }^{17}$. And if all the requirements could be reduced to a coherent minimal set of key requirements, then this would constitute a single account of computation. Premise three would then no longer follow from the preceding premises. However, premise

\footnotetext{
16 The various accounts of concrete computation offer different perspectives on what a physical computing system does. But rather than having the same extension (e.g., the operation of a UTM or a personal MacBook), these accounts end up denoting different classes of computing systems (e.g., the triviality "account" denotes all physical systems as computing, Algorithm Execution à-la Cummins includes UTMs, but excludes TMs, etc.). This makes it even more pressing to explicitly specify the appropriate account of computation.

${ }^{17}$ An overlap among requirements clearly does not imply reduction from one requirement to another. My intent here is to address a possible criticism to the effect that premise three would no longer follow as an intermediate conclusion from its preceding premises.
} 
two suggests that although some of the requirements may overlap, not all of them do. For instance, there is an overlap between the fourth key requirement implied by Turing's account (having the capacity to follow instructions) and the common requirement implied by the Algorithm Execution account. Still, other requirements such as the situatedness of the computing system (that is implied by my reconstruction of Smith's account) are not reducible to any of the other requirements examined in this paper.

Possible challenges to my conclusion might be that some of the key requirements implied by different accounts could be synthesised or that one could simultaneously subscribe to two accounts or more. The first challenge may result in sidestepping the demand to commit to an explicit account. But even if that were the case, such a synthesis would simply yield a new (possibly adequate!) account of computation. The second challenge needs unpacking. It can be interpreted in one of two ways. Firstly, it could be interpreted as subscribing to more than one account simultaneously for explaining different cognitive capacities respectively. I do not see that as a problem. There is still a need to commit to a particular account for each relevant cognitive capacity. But this could have some other consequences, such as explaining cognitive behaviour in a non-unified manner by resorting to a plethora of computational models.

Secondly, the challenge could be interpreted as subscribing to several accounts simultaneously, since cognitive explanations by nature span multiple levels. This is consistent with David Marr's (1982) three levels of analysis. For instance, we could hold that (1) cognitive computations are inherently representational. At the same time, we could also hold without being inconsistent that (2) these computations are constrained in terms of any one of the formalisms of computability, and lastly that (3) they occur in the brain, which is embodied 
and situated in the real world. This is all well and good. Still, as I have argued above, concrete computation (but perhaps not cognitive computation) could be explained without necessarily invoking any representational properties (e.g., Copeland's Algorithm Execution account). If one wishes to commit to a representational account of digital computation, since cognition is representational, one should firstly justify why computation proper is representational. Also, subscribing to an account of concrete computation and to a formalism of computability simultaneously does not introduce any conflict (this point is further discussed below).

\subsection{Concrete digital computation is far from being trivial}

For Searle (1990: pp. 25-26) the fact that TMs could be physically implemented on "just about anything" has adverse consequences: every physical system performs digital computation. Although his account could also be interpreted as dealing with FSA computation, it is not confined to FSA and is just as applicable to TMs. Searle argues that if we accept Turing's account and define computation in terms of assigning 0's and 1's, then every system is a digital computer, since it could be described in terms of 0's and 1's. Thus, as long as the system is both sufficiently complex and apprised of by a knower - it computes! This is probably not what Turing had in mind when he introduced his abstract machines (a digestive system could also be described in terms of 0's and 1's). David Chalmers (1995: pp. 395-396, 1996: pp. 331-332) adds that the implementation relation between abstract automata and physical systems is objective and is not relative to a knower. The states of Searle's wall will (almost) certainly not satisfy the relevant reliable state-transition rules, for his wall (and probably any other wall) does not possess the required causal organisation to compute ${ }^{18}$.

\footnotetext{
${ }^{18}$ Chalmers $(1995,1996)$ argues that every physical system implements a simple FSA with a single internal state, for instance. Instead he recommends using the formalism of combinatorial state automata (CSA), and argues that the states in most computational formalisms have a combinatorial structure. The conditions for implementing a given complex computation using CSAs will be typically sufficiently rigorous to block arbitrary state transition mappings à-la Searle.
} 
Fortunately, Searle's account could be resisted, for one thing, since it cannot explain certain counterfactuals, which must be true about computing systems. As Block (2002: pp. 77-78) points out, a computing system should allow all the possible computations, which the system could have performed, rather than just the one it actually performs. Suppose that Searle's wall computes " $0+1=1$ ". For this wall to be deemed a computing system, it is not enough to have the physical states corresponding to the states ' 0 ' and ' 1 ' followed by a physical state corresponding to ' 1 ' (representing the output). It must also allow all the other possible computations that could have been performed by a computing system. For instance, had the ' 0 ' input been replaced by a ' 1 ' input, the output would have been replaced by a ' 2 '.

Additionally, Searle's metaphysics of computation (SM) could give rise to some absurd consequences. For example, if we accepted SM and the theory of complexity (TC) then some unavoidable absurd consequences would follow. One surprising consequence is that there could be a knower arguing that the complexity classes $P^{19}$ and $N P^{20}$ are equivalent, since Searle argues that computation is knower-relative. He could argue that a big enough wall could compute the Travelling Salesperson Problem (TSP) ${ }^{21}$ in P. This would be the case under a description in which each state transition corresponded to adding an edge to the optimal route, and no more than (the order of) $\mathrm{n}$ such state transitions were needed for constructing the optimal route through n cities. The knower could simply "know" what the optimal route is (for a given input), for SM does not support counterfactuals about computation and there exists some arbitrary description under which the wall computes TSP

\footnotetext{
${ }^{19}$ An algorithm is said to be $P$ or polynomial time if its execution time is upper bounded by a polynomial in the size of the input for the algorithm, i.e., $T(n)=\mathrm{O}\left(n^{k}\right)$ for a constant $k \_\mathrm{N}$

${ }^{20}$ Formally, a language $\mathrm{L}$ is in the class NP (non-deterministic polynomial time) iff $\mathrm{L}$ is accepted by a nondeterministic TM, which operates in polynomial time (Karp 1972: p. 91).

${ }^{21}$ TSP involves determining whether there is some possible route through the nodes (cities) in a graph that enables each city to be visited exactly once. The question is whether this graph has a set of edges that connect the nodes into a single cycle with each node appearing exactly once. All ways to solve this problem require trying essentially all cycles and computing their total weight (Hopcroft et al 2001: pp. 419-420).
} 
in P. And if TSP turned out to be in P, it would follow that $\mathrm{P}=\mathrm{NP}$ (Hopcroft et al 2001: pp. 419-423). However, this consequence is precipitated, as it is one of the deepest open questions in mathematics and computer science. If we reject this consequence as unacceptable, then SM must be rejected as false (stipulating that TC is accepted to be true).

Although Putnam-triviality escapes some of the problems that Searle-triviality faces, it still faces some serious problems concerning physical computation. Putnam's account focuses more on the mathematical realm and ignores some physical constraints that unavoidably apply to concrete computation. A central principle in thermodynamics states that a finite amount of space has finite entropy. Physical systems with finite entropy can only perform a finite number of operations (Penrose 1989: pp. 391-417). It follows from these two constraints that any finite physical system (such as ordinary open systems) cannot perform infinitely many computations. So, Putnam-triviality can be resisted in the physical world.

Moreover, his principles of continuity and of non-cyclical behaviour only alleviate some of the problems for trivial computation. Physical computing systems may exhibit spatial discontinuities and yet we can determine which computation has been performed when an error occurs. Provided that the physical conditions under which the error has occurred are known, a theory of error allows us to analyse it. Putnam does indeed exclude physical systems that exhibit spatial discontinuities or chaotic behaviours. But, even if the physical conditions are known, Putnam-triviality does not allow us to predict in some possible systems whether they will exhibit such behaviours, and violate the principle of continuity. If a physical system $\mathrm{S}$ violated that principle, then $\mathrm{S}$ would not compute $\mathrm{F}$. If $\mathrm{S}$ did not violate the principle of continuity, we still cannot predict whether S trivially computes F. Thus, Putnam-triviality 
faces a Kripke-Wittgenstein problem ${ }^{22}$ (Buechner 2008: p. 131).

Furthermore, the principle of non-cyclical behaviour also excludes many control constructs, which are commonplace in programming languages, thus excluding many computations. It prohibits a physical system from having a duplicated physical state and entering the same maximal state twice ${ }^{23}$. But this simply rules out computations, which contain looping on a particular condition or GOTO statements. For example, suppose that a physical system $S_{1}$ actualises a computation that contains a loop statement, which keeps running until a signal is received from another system $S_{2}$. The signal from $S_{2}$ is the exit condition for the loop statement in $\mathrm{S}_{1}$. If $\mathrm{S}_{1}$ does not change its computational state whilst running the loop statement, it will be excluded by the principle of non-cyclical behaviour, since $\mathrm{S}_{1}$ reenters the same maximal state.

Additionally, Putnam's account does not support some counterfactuals that are relevant to concrete computation. Putnam considers the class of counterfactuals of the form "if a physical system had not been in state A, it would not have transited to state B". But what is required is not just that the state A is followed by state B in a given time period, as he suggests. Rather, there must exist a reliable counterfactual supporting relation between the states. It must obtain that if the physical system were to be in state A, it would transit to state B. This condition must also be satisfied for every transition, which is specified in the machine table, and not only for those whose antecedent states occur in a certain time period. Putnam's account does not meet this stronger requirement (Chalmers 1995: pp. 398-399).

\footnotetext{
${ }^{22}$ A Kripke-Wittgenstein problem is an extension of Ludwig Wittgenstein's rule-following problem. Saul Kripke asks what determines objectively whether one means plus rather than quus. He introduces quus as the addition function (typically known as plus) for all sums below a certain computed threshold, but diverges for higher numbers. Traditional usage of plus soon becomes susceptible to many quus-like interpretations. So the application of plus is no longer governed by a strictly defined rule.

${ }^{23}$ Prohibiting a system from entering the same computational state twice does not confine Putnam's account to FSA computation, but it does render it inapplicable to cyclic FSA, cyclic TMs and those that must use their tape.
} 
On Smith's view, the trivialisation consequence of Turing's account is interesting indeed: it is reduced to a physical theory, specifically "a mathematical theory of causality" (Smith 2002: p. 42; 2010: 23). He argues that Turing's theory is not and can never be successful in deriving even an abstract notion of effective computability and fails as a theory of computation. Computation is constrained by and substantiated by physical connections between the computing system and the physical world. Classical computability theory is, according to Smith, a general theory of the physical world: how hard it is for one physical configuration to change into another by means of either scanning and writing symbols or some other finite physical processes (Smith 2010: p. 27). Instead, on a reconstructive reading of Smith, a computing system has to satisfy the three above requirements, which imply that it is representational as well as effective.

Additionally, Smith (2010: pp. 29-31) asserts that UTMs as a means of analysing equivalences of various computing systems are at best misleading. UTMs obscure our understanding of the powers and physical limitations of computing systems. This also leads to the unjustified assumption that TM controllers, CPUs etc. are concrete entities, whilst instruction tables, programs and so on are not. But once this assumption is rejected and programs too are acknowledged as being concrete configurations of marks or symbols, the conceptual boundary between CPUs and programs begins to fade. By the same token, when analysing the equivalence of one TM to another, not only should the "machines" be compared, but so should the respective programs executed on these machines.

On the other hand, Copeland (1996: p. 335) maintains that classical computability theory provides necessary conditions for distinguishing computing systems from noncomputing systems. To uphold the sufficiency of Turing's theory, he argues that a distinction 
should be drawn between standard and nonstandard interpretations of computation (ibid: pp. 346-348). On a nonstandard interpretation of a theory the intended meanings of the terms of the theory are not respected. For instance, on a nonstandard interpretation (as Searle's) of the axiomatic specification of the physical system and the computation it performs, it might turn out that walls, clocks and buckets of water are indeed computing systems.

Also, on a nonstandard interpretation à-la Searle's, all the alleged computational activity occurs outside the wall, whilst interpreting the molecular movements of the wall as computational and assigning the labelling scheme ex post facto. Searle's labelling scheme is incomplete, for he imposes a scheme that only applies to a particular time interval (rather than being applicable throughout the lifetime of the computing wall). Additionally, future computational states cannot be predicted and counterfactuals are not supported. When extending Turing's account by adding Copeland's honest model requirement, which preserves the non-arbitrariness of computation, these difficulties are addressed. Copeland's extended Turing account then provides both necessary and sufficient conditions for distinguishing computing systems from non-computing systems (Copeland, personal communication).

Although some of the key requirements identified above may overlap, they still reveal sufficient dissimilarity between the accounts examined. Even when excluding Putnam and Searle's trivialisation of digital computation, the three remaining accounts imply several unique requirements for a physical system to perform digital computation. The first three key requirements implied by Turing's account are the basis for any actual algorithmic computation. An actual algorithmic computation is a spatiotemporal activity, as opposed to just being a mathematical entity (Israel 2002: p. 190). And any system, in the course of this activity, which cannot distinguish between different symbols, cannot write symbols to 
memory or change its states, is certainly not running an efficacious algorithm.

Moreover, the four requirements, which are implied by Turing's account, overlap with the prime requirement of the Algorithm Execution account. Turing's fourth key requirement (the capacity to follow instructions) simply ensures that the system can indeed execute (or act in accordance with) an algorithm. Some may argue that only UTMs execute programs (or algorithms), but not standard TMs (cf. Piccinini 2008a). Still, this simply calls for a further distinction between a program-controlled computing system (i.e., a special purpose TM) and a stored-program computing system (i.e., a general purpose UTM). The former is hardprogrammed (or hardwired) for a special purpose computation by modifying the machine head's internal wiring (e.g., by means of a plug-board arrangement). The latter is softprogrammed by inscribing the machine's instructions onto its tape (Copeland 1997: p. 691).

But the Algorithm Execution account adds further requirements, which are not explicitly required by Turing's account. In the spirit of Turing, Copeland (1997: p. 696) defines an algorithm as "any collection of rules or instructions that determines the behavior of a computing machine". He introduces the existence of a labelling scheme and the honest model requirements to successfully block absurd consequences that follow from trivialising the notion of digital computation (Copeland 1996).

Moreover, Smith $(2002 ; 2010)$ insists on further requirements that are usually ignored by traditional accounts of computation. On his view, computing systems are essentially situated in their environment, rather than merely operating in isolation, where their situatedness is merely contingent. Though the behaviour of computing systems is indeed effective, it is not limited to the physical boundaries of the system. Incidentally, Wells' view bears resemblance to Smith's in relation to the situatedness of computing systems. Wells also 
argues that UTMs (and computers) are embedded in an environment, which constitutes part of the TM's architecture (Wells 1998: p. 280). According to Smith, computing systems are representational and as such they stand in semantic relations to distal states of affairs.

\subsection{Accounts of concrete computation and formalisms of computability}

Finally, there is no question whether mathematical formalisms of computability are adequate analyses of computability, but they are of the wrong kind to explain concrete computing systems. In what follows, I argue that formalisms of computability may provide the mathematical tools required for determining the plausibility of computational level theories. Yet, any particular formalism does not specify the relationship between abstract and concrete computation. It is at the physical level the algorithm is specified and bound by the implementing physical system. So stipulating that any complete account of a physical phenomenon must also consider its physical implementation, an explicit account of concrete computation has to be specified for a complete account of computing systems.

This argument follows almost immediately from Marr's three levels of analysis. If his explanatory model is interpreted as a top down methodology ${ }^{24}$, then the top level provides an extensional account of what is computed, the middle level specifies how it is computed, and the bottom level describes the actual implementation. The top/computational level characterises the mathematical (or cognitive) input/output function being computed. The middle/algorithmic level specifies how the system computes this function and is consistent with various formalisms of computability. The implementational/bottom level shows how the system works in terms of the physical system and this is certainly where accounts of concrete

\footnotetext{
${ }^{24}$ This conventional interpretation has had its fair share of criticisms (see for example, Bell et al 2001: pp. 209212; Dennett 1998: pp. 249-260; Shagrir 2010). For my purposes, suffice to say that Marr identifies the top level as computational. But it is his algorithmic level that is on a par with formalisms of computability, and the implementational level that describes physical computation.
} 
computation fit in comfortably.

Indeed, formalisms of computability may provide the mathematical tools required for evaluating the plausibility of computational level theories. Since our cognitive capacities are constrained by the fact that we are finite systems with limited resources for computation, some argue that classical computability theory and complexity theory could assist cognitive science by providing theoretical constraints on the set of feasible computational level theories. An underlying assumption of this view is that a cognitive capacity involves the effective computation of a specific input/output function F (i.e,, a set of ordered pairs), which given an initial/input state $\mathrm{i}$, realises a final/output state $\mathrm{o}=\mathrm{F}(\mathrm{i})$ as specified by function $\mathrm{F}$ : $\mathrm{I} \rightarrow \mathrm{O}$. If a computational level theory of a cognitive capacity is assumed veridical, then further attempts can be made by scientists to analyse it at the next levels of abstraction, which Marr called the algorithmic and the implementational levels (van Rooij 2008: pp. 939-941).

Once a particular mathematical formalism was chosen to evaluate the feasibility of a computational level theory, the next level of abstraction has to be taken into account, namely the physical level. Each one of the mathematical formalisms of computability provides a definition of the set of allowable operations used to compute functions and their associated computational costs by implication. So in order to determine the computational resources that are required, the particular formalism needs to be specified (though some complexity distinctions, such as between P and NP running time are insensitive to the exact formalism of computability). But even then, the particular formalism does not specify the relationship between abstract and concrete computation (in Chalmers 1995, he argues that it is a relation of isomorphism between the formal structure of the former and the causal structure of the latter).

The next task then is choosing the appropriate computability formalism for an analysis 
at the algorithmic level. Daniel Dennett (1998: p. 232) suggests that an analysis at the computational level "might be nothing more that the demand for enough rigour and precision to set the problem for the next level down, Marr's algorithmic level". If we are to agree with Marr that problems must first be faced at the computational level (ibid: p. 231), then we cannot avoid questions regarding algorithmic and concrete computation at the next levels. Some formalisms of computability, such as TMs and lambda calculus, specify the algorithm to be employed to compute the computational level function, whereas others, recursive functions, for instance, do not. The appropriate formalism provides an algorithmic level explanation of how ordered pairings at the computational level are computed. Still, there could be many algorithms, which correspond to any particular computational level function, that could be in turn implemented in any number of ways.

So, it is only at the implementational level that the algorithm has been specified and constrained by the particular physical system. This is where an account of concrete computation has to be specified explicitly, as I have argued above. The appropriate account of concrete computation could be used to bind the algorithmic level (e.g., TM, lambda calculus, etc.) and the physical level together to explain the actual computation taking place. This also shows that there is no conflict in subscribing simultaneously to both a formalism of computability and an account of concrete computation.

Whereas the Algorithm Execution account and Smith's reconstructed account also make provisions for the algorithmic level, Turing's analysis clearly does not account for spatiotemporal considerations. The Algorithm Execution account à-la Copeland tries to meet the objection to Turing's analysis as necessary but insufficient (Copeland 1996: p. 335). He leaves Turing's analysis at the algorithmic level unchanged, but extends it to be closely coupled with the implementational level in considering the formal description of the 
computing system, the algorithm executed and the supporting architecture. Smith's reconstructed account puts most emphasis on the algorithmic and implementational levels in explaining the crossing of boundaries between the abstract algorithms and the physical realworld computing systems. Lastly, Turing's analysis is aligned with the algorithmic level in accordance with the four requirements specified above. But his analysis of the implementational level is lacking, for it overlooks spatiotemporal considerations.

This point was recognised by Gandy (1980: pp. 124-125), who argued that concrete computation requires an independent analysis that considers the limits of physical computation. He postulated explicit physical constraints on computing systems, namely a lower bound on the size of distinguishable atomic components of the computing system, and an upper bound on velocity of propagation of information. These restrictive conditions are motivated by purely physical considerations (Sieg 2008: p. 147): the uncertainty principle of quantum mechanics justifies a lower bound on the size of atomic components, and the theory of special relativity yields an upper bound on signal propagation ${ }^{25}$. Interestingly, Smith (2008), who criticises Turing's theory as being "a mathematical theory of causality", praises Gandy for recognising the importance of physical constraints in computing systems and showing that absolute computability results depended on the character of the implementing physical system.

\section{Conclusion}

A new account of concrete computation was not offered here. Two arguments were

\footnotetext{
${ }^{25}$ Copeland and Shagrir (2007) object to the claim that all the finiteness constraints are motivated by physical considerations, as there are physical systems that satisfy Gandy's principles, are finite in the physical sense, but are (the controversial) hypercomputers. Their objection is motivated by an ambiguity in the term 'deterministic' (ibid: p. 228). Some hypercomputers are not 'Gandy deterministic', for there is no such thing as the previous state that uniquely determines the configuration of its halting state, yet they invoke no random or stochastic elements.
} 
given instead. The main argument was that well-known accounts of concrete computation entail sufficiently distinct requirements for a physical system to compute, justifying the demand that one commits to a particular account when employing the notion of concrete computation. The second one was that whilst mathematical formalisms of computability may play an informative role in cognitive science, they do not specify the relationship between abstract and concrete computation, and are of the wrong kind to explain the phenomenon of concrete computation.

But surprisingly despite the apparent straightforwardness of these arguments, all too often their implied morals are ignored by philosophers and cognitive scientists. The notions of computation simpliciter and digital computation in particular are employed without much awareness of what they mean exactly. At times, these accounts are even used interchangeably as though they were equivalent (when they are not even extensionally equivalent). No less problematic is the appeal to formalisms of computability (most commonly to TMs) when the explanandum is a physical phenomenon and its physicality is central to its explanans. If we take cognition to be a physical phenomenon and try to explain it computationally, we should say explicitly what we mean by computation.

\section{Acknowledgements}

Thanks are due to Gualtiero Piccinini and David Chalmers for useful comments on earlier drafts of this paper. I have greatly benefited from discussions with Oron Shagrir, Joseph Agassi and Jack Copeland on various aspects examined in the paper and for that I am thankful. I would also like to thank several anonymous referees for the Journal of Logic, Language and Information for their invaluable comments that helped reshape and improve this paper. I am especially indebted to Phillip Staines for his fruitful criticism and ongoing support. An early version of this paper was presented at the 2010 AAP conference in Sydney, Australia. All the people mentioned above contributed to the final draft of the paper, but I am responsible for any remaining mistakes. 


\section{References}

- Agassi, J. (unpublished). The Turing Test.

- Bell, P., Staines, P. J., Mitchell, J. (2001). Evaluating, doing and writing research in psychology. Sage publication.

- Block, N. (2002). Searle's Arguments Against Cognitive Science. In J. Preston and M. Bishop (eds.) Views into the Chinese Room. pp. 70-79. Oxford: Oxford University Press.

- Buechner, J. (2008). Gödel, Putnam, and Functionalism. Cambridge: MIT Press.

- Chalmers, D. (1995). On implementing a computation. Minds and Machines, 4, pp. 391402.

- Chalmers, D. (1996). Does a rock implement every finite-state automaton? Synthese, 108, pp. 309-333.

- Chrisley, R. L. (1995). Why everything doesn't realize every computation. Minds and Machines, 4, 403-420.

- Copeland, B. J. (1996). What is computation? Synthese, 108, pp. 335-359.

- Copeland, B. J. (1997). The broad conception of computation. The American Behavioral Scientist, 40, pp. 690-716.

- Copeland, B. J. (2003). Computation. In L. Floridi (Ed.) The Blackwell guide to the philosophy of computing and information. pp. 3-17. Wiley-Blackwell.

- Copeland, B. J. and Shagrir, O. (2007). Physical computation: how general are Gandy's principles for mechanisms? Minds and Machines, 17, pp. 217-231.

- Cummins, R. (1977). Programs in the explanation of behaviour. Philosophy of Science, 44, pp. 269-287.

- Cummins, R. (1989). Meaning and Mental Representation. Cambridge: MIT Press.

- Cummins, R. (1996). Systematicity. The Journal of Philosophy, 93, pp. 591-614. 
- Davis, M. (1958). Computability and unsolvability. NY: McGraw-Hill.

- Dennett, C. D. (1998). Brainchildren: essays on designing minds. Cambridge: MIT Press.

- Fodor, J. A. (1975). The language of thought. Cambridge: Harvard University Press.

- Fresco, N. (2008). An analysis of the criteria for evaluating adequate theories of computation. Minds and Machines, 18, pp. 379-401.

- Fresco, N. (2010). Explaining computation without semantics: keeping it simple. Minds and Machines, 20, pp. 165-181.

- Gandy, R. (1980). Church's thesis and principles for mechanisms. In J. Barwise, H. J. Keisler \& K. Kunen (Eds.), The Kleene symposium. Amsterdam: North-Holland.

- Hopcroft, J. E., Motwani, R. and Ullman, J. D. (2001). Introduction to automata theory, languages and computation. Addison Wesley: $2^{\text {nd }}$ edition.

- Israel, D. (2002). Reflections on Gödel's and Gandy's reflections on Turing's thesis. Minds and Machines, 12, pp. 181-201.

- Karp, R. M. (1972). Reducibility among combinatorial problems. In R. Miller \& J. Thatcher (Eds.), Complexity of computer computations, pp. 85-104. New York: Plenum.

- Kleene, S. C (2002). Mathematical logic. New York: Dover.

- Marr, D. (1982). Vision: A computational investigation into the human representation and processing visual information. NY: Freeman \& Company.

- Nelson, R. J. (1982). The logic of mind. Dordrecht: Reidel.

- Newell, A. and Simon, H. A. (1976). Computer science as an empirical enquiry: symbols and search. Communications of the ACM, 19: pp. 113-126.

- Parikh, R. (1998). Church's theorem and the decision problem. In E. Craig (Ed.) Routledge Encyclopedia of Philosophy, 10, pp. 349-351. London: Routledge.

- Penrose, R. (1989). The Emperor's new mind. London: Oxford University Press.

- Piccinini, G. (2007). Computing mechanisms. Philosophy of Science, 74, 501-526. 
- Piccinini, G. (2008a). Computation without representation. Philosophical studies, 137, pp. 205-241.

- Piccinini, G. (2008b). Computers. Pacific Philosophical Quarterly, 89, pp. 32-73.

- Piccinini, G. \& Scarantino, A. (2011). Information processing, computation, and cognition. Journal of Biological Physics, 37, pp. 1-38.

- Putnam, H. (1988). Representation and reality. Cambridge: The MIT Press.

- Pylyshyn, Z. W. (1984). Computation and cognition: toward a foundation for cognitive science. Cambridge MA: MIT Press.

- Searle, J. R (1990). Is the Brain a Digital Computer? Proceedings and Addresses of the American Philosophical Association, 64, pp. 21-37.

- Seligman, J. (2002). The scope of Turing's analysis of effective procedures. Minds and Machines, 12, pp. 203-220.

- Shagrir, O. (1999). What is computer science about? The Monist, 82, pp. 131-149.

- Shagrir, O. (2010). Marr on computational-level theories. Philosophy of Science, 77, pp. $477-500$.

- Sieg, W. (2008). Church without dogma: Axioms for Computability. In S.B. Cooper, B. Löwe, \& S. Andrea (Eds.) New computational paradigms: pp. 139-152.

- Smith, B. C. (1996). On the origins of objects. Cambridge, MA: MIT Press.

- Smith, B. C. (2002). The foundations of computing. In M. Scheutz (Ed.) Computationalism: new directions. pp. 23-58. Cambridge: MIT Press.

- Smith, B. C. (2008). Rehabilitating representation. Paper presented at the spring 2008 colloquia at the Center for cognitive science, University at Buffalo, State University of New York.

- Smith, B. C. (2010). Age of Significance: introduction. Retrieved May 3, 2010, from $\underline{\text { www.ageofsignificance.org }}$ 
- Smith, B. C. (unpublished). Formal symbol manipulation: ontological critique. Expected to be published in 2011 at http://www.ageofsignificance.org

- Soare, R. (1996). Computability and recursion. The Bulletin of Symbolic Logic, 2, pp. 284-321.

- Soare, R. (2009). Turing oracle machines, online computing, and three displacements in computability theory. Annals of Pure and Applied Logic, 160, pp. 368-399.

- Thelen, E. and Smith, L. B. (1994). A dynamical systems approach to the development ofcognition and action. Cambridge: MIT press.

- Turing, A. M. (1936). On computable numbers, with an application to the Entscheidungsproblem. Proceedings of the London Mathematical Society, Series 2, 42, pp. $230-265$.

- Van Gelder, T. and Port, R. F. (1995). It's about time: an overview of the dynamical approach to cognition. In van Gelder, T. and Port, R. F. (Eds.) Mind as motion. Cambridge: MIT Press.

- van Rooij, I. (2008). The tractable cognition thesis. Cognitive Science, 32, pp. 939-984.

- Wells, A. J. (1998). Turing's analysis of computation and theories of cognitive architecture. Cognitive Science, 22, pp. 269-294. 